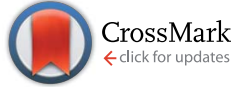

Cite this: J. Mater. Chem. B, 2015, 3, 2635

Received 12th September 2014

Accepted 13th February 2015

DOI: $10.1039 / \mathrm{c} 4 \mathrm{tb} 01520 \mathrm{~g}$

www.rsc.org/MaterialsB

\section{Development of bioconjugated dye-doped poly(styrene-co-maleimide) nanoparticles as a new bioprobe}

\author{
A. Swanepoel, ${ }^{\text {ab }}$ I. du Preez, ${ }^{a}$ T. Mahlangu, ${ }^{a}$ A. Chetty ${ }^{a}$ and B. Klumperman ${ }^{\star b}$ \\ Fluorescent dye-doped poly(styrene-co-maleimide) nanoparticles were synthesised and functionalized \\ with amine groups and avidin and the dye-loading was varied. The nanoparticles showed great potential \\ as bioprobes when conjugated with biotinylated antibodies.
}

Dye-doped nanoparticles represent highly sensitive labelling devices as each particle potentially contains thousands of dye molecules localized inside a protective shell. When functionalized with receptor molecules such as antibodies, peptides, or lipids, these nanoparticles cluster around target cells, creating localized amplified fluorescence signals. ${ }^{1}$ Silica based dyedoped nanoparticles have been extensively developed and tested for detection of bacteria. ${ }^{1-5}$ Fluorescent nanocrystals or quantum dots (QDs) have also been used for signal amplification and detection of microorganisms. ${ }^{6-9}$ QDs display high quantum yields, but suffer from potential toxicity due to leaching of heavy metal ions from the particles. ${ }^{10} \mathrm{~A}$ method was published for the simple one-step synthesis of large volumes of nano-sized poly(styrene-co-maleimide) (PSMI) nanoparticles via the imidization of poly(styrene-co-maleic anhydride) (PSMA). ${ }^{11}$ Compared to this simple preparation method, the synthesis of silica nanoparticles according to the classic Stöber method is highly laborious. The aim of the present work was to study the use of PSMI nanoparticles as a bioprobe by encapsulating a hydrophobic fluorescent dye, Exalite 613, and functionalizing the surface of the nanoparticles with amine groups and avidin in order to facilitate the orientated attachment of anti-Escherichia coli (E. coli) biotinylated antibodies. Dye doping was studied by fluorescence spectroscopy. Avidin attachment to nanoparticles was quantified via a biotin(5-fluorescein) (B5F) conjugate according to a titration method. ${ }^{4}$ Finally, the attachment of fluorescent nanoparticles to $E$. coli bacteria was visualized via fluorescence microscopy.

High molecular weight PSMA was obtained from Polyscope (Geleen, The Netherlands) with a weight average molecular weight of $80000 \mathrm{~g} \mathrm{~mol}^{-1}(D \cong 2)$ and a maleic anhydride content of $26 \mathrm{wt} \%$. Exalite 613 (Perylene Red) dye was purchased from Exciton Inc (USA). Formaldehyde (37\% aqueous solution)

${ }^{a}$ Polymers and Composites, Materials Science and Manufacturing, Council for Scientific and Industrial Research, Pretoria, South Africa

${ }^{b}$ Department of Chemistry and Polymer Science, Stellenbosch University, Stellenbosch, South Africa. E-mail: bklump@sun.ac.za and 1,4-diaminobutane (DAB) were obtained from Sigma. 1-[3-(Dimethylamino)propyl]-3-ethylcarbodiimide methiodide (EDC) (Aldrich), $N$-hydroxysuccinimide (NHS) (Aldrich), bovine serum albumin (BSA) (Fluka), rabbit anti Escherichia coli:biotin antibodies (AdB Serotec) and avidin from egg white were used for bioconjugation, as well as Tween 20 (Aldrich), trisma hydrochloride (Tris-HCl) (Aldrich) and biotin(5-fluorescein) (B5F) (Sigma). Phosphate buffer saline (PBS) at $0.1 \mathrm{M}(1 \times)$ was prepared and used for nanoparticle preparation and for preparation of E. coli suspensions.

Dye-doped PSMI nanoparticles were prepared via the imidization of PSMA in a $250 \mathrm{~mL}$ picoclave reactor (Büchi, Switzerland). The reactor was charged with PSMA (20 g), deionised water $(100 \mathrm{~mL})$, ammonium hydroxide solution $(5 \mathrm{~mL}, 25 \%)$, and Exalite 613 dye $(5 \mathrm{mg}, 7.5 \mathrm{mg}$ or $10 \mathrm{mg})$. Imidization was allowed to proceed at a bath temperature of $180^{\circ} \mathrm{C}$, with stirring at $1000 \mathrm{rpm}$, for 6 hours. After completion of the reaction and cooling of the latex, nanoparticle dispersions were dialysed with

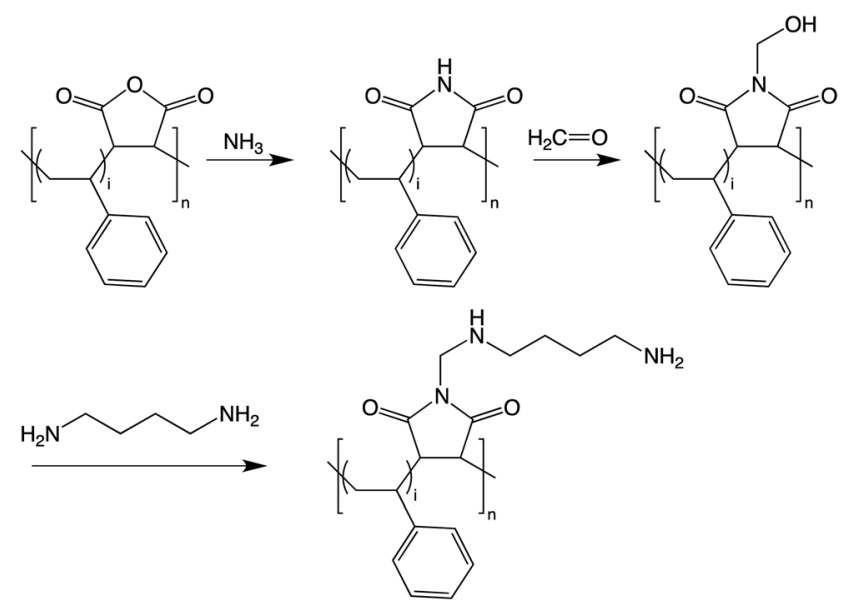

Scheme 1 Reaction scheme for the imidization of PSMA to PSMI and the surface functionalization of the PSMI with formaldehyde and DAB. 


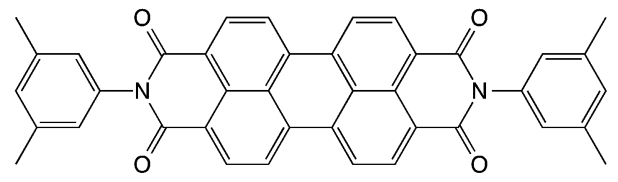

Fig. 1 Structure of Exalite 613 red laser dye.

a 10000 molecular weight cut off snake skin dialysis tube to remove unreacted molecules. PSMI nanoparticles were consecutively functionalized with molar excesses of formaldehyde and $\mathrm{DAB}$, and dialysed after each step, in order to introduce reactive amine groups onto the nanoparticle surfaces. All reactions were performed in the liquid media of the nanoparticle dispersion. The proposed reactions for nanoparticle synthesis and surface functionalization are shown in Scheme 1.

The molecular structure of Exalite 613 as given by the supplier is shown in Fig. 1. Exalite 613, apart from its excellent stability and fluorescent intensity, is also hydrophobic in nature. It was postulated that, upon addition of a hydrophobic dye to the aqueous suspension in the reactor, the dye would preferably be encapsulated inside the nanoparticles rather than remain in the aqueous media. Interaction between dye and PSMI was thus expected to be either physical in nature, or as a result of $\pi-\pi$ stacking between the aromatic rings of the styrene and the dye molecules.

Avidin was attached to amine-functionalized dye-loaded NPs via a published method using EDC and NHS. ${ }^{12}$ It was postulated that the amine groups functionalised onto the nanoparticles would form amide bonds with carboxylic acids present on the avidin molecule, a process that would be mediated via carbodiimide chemistry. The reaction mechanism is shown in Scheme 2. Nanoparticle dispersions were reacted with EDC, NHS and avidin in a $0.05 \%$ PBS-Tween 20 solution for 24 hours at $37^{\circ} \mathrm{C}$ while being gently agitated. The nanoparticles were washed and resuspended in a Tris- $\mathrm{HCl}$ buffer with a $0.05 \%$ BSA content and incubated for 1 hour at $25^{\circ} \mathrm{C}$ under gentle agitation to block any unreacted sites. The avidin modified nanoparticles were resuspended in PBS-Tween with rabbit anti-E. coli biotinylated antibodies at $37^{\circ} \mathrm{C}$ for $1 \mathrm{hour}$, and after washing was resuspended in $1 \times$ PBS-Tween $(0.05 \%)$ solution for use with bacteria.

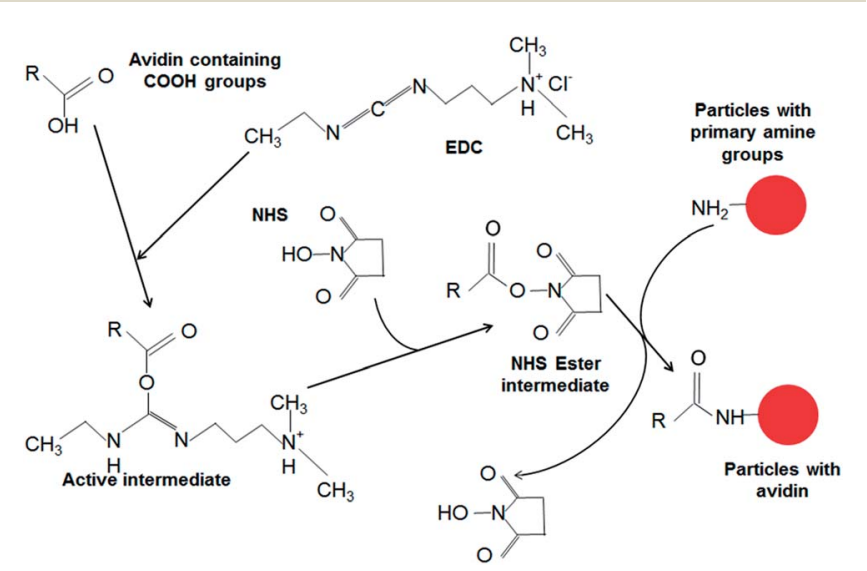

Scheme 2 Proposed reaction mechanism for the carbodiimide assisted bioconjugation of the PSMI nanoparticle surfaces with avidin.
Quantitative detection of avidin immobilized onto the nanoparticle surfaces was performed indirectly via quenching of the inherent fluorescence of a B5F conjugate by avidin present in the liquid supernatant separated from the bioconjugated nanoparticles. ${ }^{4}$ Titration assays were performed with both the nanoparticle supernatant and pure avidin, and the fluorescence of the solutions measured with a spectrofluorometer at $\lambda_{\mathrm{ex}} / \lambda_{\mathrm{em}}=485 \mathrm{~nm} / 528 \mathrm{~nm}$.

E. coli $\mathrm{K}-12$ suspensions were prepared via serial dilutions and contacted with $0.02 \mathrm{mg}$ of dye-doped nanoparticles for 1 hour under gentle agitation. The samples were also stained with SYTO 9 green fluorescent nucleic acid stain and viewed with a fluorescence confocal microscope. E. coli cells contacted with dye-doped nanoparticles were added to $1 \mathrm{~mL}$ of a $2.5 \%$ glutaraldehyde solution and fixed for an hour for sectioning and imaging via SEM.

The imidization of PSMA to PSMI as well as dye-doping and surface functionalization of the PSMI nanoparticles was characterized via ATR-FT-IR spectroscopy. The FT-IR spectra of pristine PSMI, dye-doped PSMI and pure Exalite 613 dye are given Fig. 2. Successful imidization of PSMA is evidenced by the appearance of characteristic peaks at $1709 \mathrm{~cm}^{-1},(\mathrm{C}=\mathrm{O}$ or $\mathrm{N}-\mathrm{C}=\mathrm{O}$ stretch, imide I), $1345 \mathrm{~cm}^{-1}$ and at $1177 \mathrm{~cm}^{-1}$ (imide II and imide III bending respectively), all indicative of imide groups. Comparison of the FT-IR spectra of the pristine and the dye-doped PSMI nanoparticles showed no differences in characteristic absorption peaks detected. Surface functionalization with an excess of formaldehyde yielded a new characteristic peak at $1400 \mathrm{~cm}^{-1}$, and this peak increased in intensity after the addition of the diamine compound, as indicated in Fig. 3. The appearance of this peak was attributed to the additional $\mathrm{CH}_{2}-\mathrm{OH}$ linkages introduced onto the NPs after formaldehyde functionalization and additional $\mathrm{CH}_{2}$ linkages introduced after amine surface functionalization. Amine functionalization resulted in the appearance of a significant peak at $1555 \mathrm{~cm}^{-1}$, indicative of primary $\mathrm{N}-\mathrm{H}$ bending. The appearance of this peak confirmed successful amine surface functionalization (indicated by arrows on the graph).

The size and morphology of the pristine and dye-doped PSMI NPs characterized via TEM displayed a narrow size range

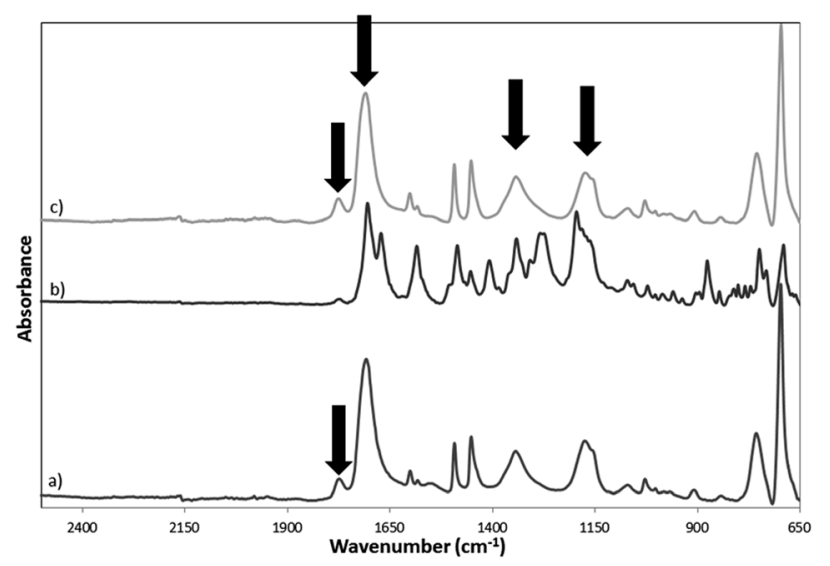

Fig. 2 FT-IR absorption spectra of (a) pristine PSMI nanoparticles, (b) pure reference Exalite 613 dye and (c) dye-doped PSMI nanoparticles. 


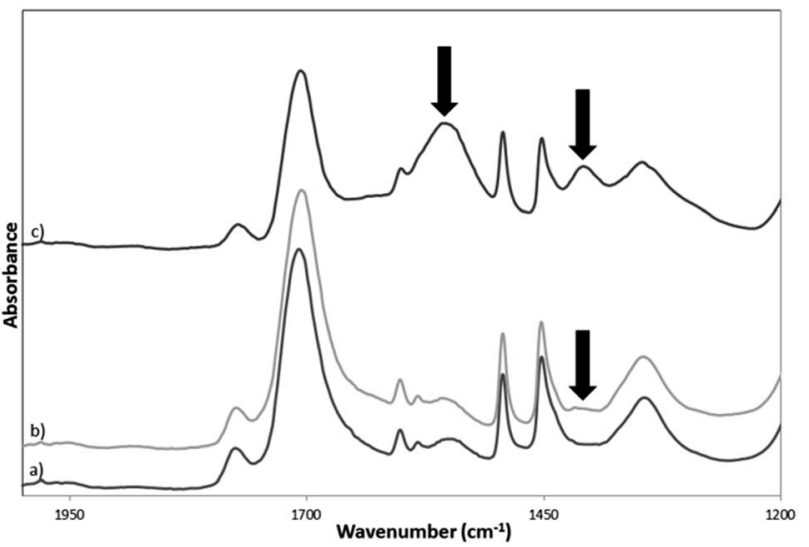

Fig. 3 FT-IR absorption spectra of (a) dye-doped PSMI nanoparticles, (b) dye-doped PSMI nanoparticles functionalized with formaldehyde and (c) formaldehyde-functionalized nanoparticles reacted with DAB.

distribution with average particle sizes of between 20 and $50 \mathrm{~nm}$, as shown in Fig. 4. Surface functionalization with formaldehyde and DAB as well as dye-doping did not alter the size or morphology of the particles when compared to the pristine nanoparticles.

The glass transition temperature $\left(T_{\mathrm{g}}\right)$ of the PSMI nanoparticles was determined via differential scanning calorimetry (DSC). The $T_{\mathrm{g}}$ of the pristine PSMI NPs was found to be $179^{\circ} \mathrm{C}$, which is in good agreement with the published value of $181^{\circ} \mathrm{C}$ for PSMI with a $26 \%$ maleimide content. ${ }^{11}$ Dye-doped PSMI nanoparticles showed a $T_{\mathrm{g}}$ of $183{ }^{\circ} \mathrm{C}$. The effect of surface functionalization on the $T_{\mathrm{g}}$ of dye-doped nanoparticles is shown in Table 1.
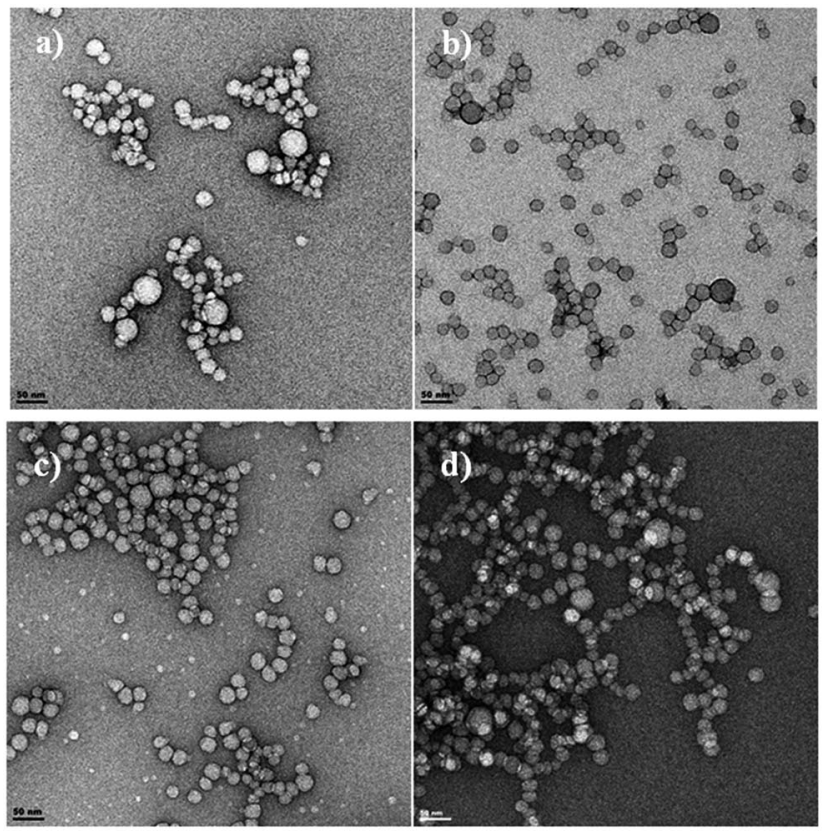

Fig. 4 Transmission electron micrographs of pristine PSMI nanoparticles (a) before and (b) after surface functionalization, as well as dye-doped PSMI nanoparticles (c) before and (d) after surface functionalization.
Table 1 Effect of surface functionalization on $T_{\mathrm{g}}$ of dye-doped PSMI nanoparticles

\begin{tabular}{lc}
\hline Sample & $T_{\mathrm{g}}\left({ }^{\circ} \mathrm{C}\right)$ \\
\hline PSMI nanoparticles & $183 \pm 0.1$ \\
$\begin{array}{l}\text { Nanoparticles functionalized with } \\
\text { formaldehyde }\end{array}$ & $190 \pm 0.1$ \\
Nanoparticles functionalized with DAB & $190 \pm 0.9$
\end{tabular}

For the surface-functionalized nanoparticles (with formaldehyde and subsequently DAB), the $T_{\mathrm{g}}$ increased significantly to $190{ }^{\circ} \mathrm{C}$ when compared to the non-functionalized PSMI nanoparticles. This increase could be explained by inter- and intramolecular interactions between the newly introduced hydroxyl group terminated structure that effectively replaced the $\mathrm{H}$ atom of the maleimide ring. This is in spite of the disappearance of the strong hydrogen-bonding $\mathrm{N}-\mathrm{H}$ of the original maleimide as a result of functionalization. This increase also means that the nanoparticles are always far below their $T_{\mathrm{g}}$ and will act as rigid glassy materials.

In order to assess the stability of the nanoparticle dispersion, zeta-potentials were measured via photon correlation spectroscopy. The pure PSMI nanoparticles possessed high negative zeta-potentials of $-67.3 \pm 1.3 \mathrm{mV}$ (at in situ $\mathrm{pH}$ of 7.1 ), corresponding well with previously reported values ${ }^{\mathbf{1 1}}$ and indicating good particle stability. These high negative surface charges were not affected by dye loading, with a charge of $-67.8 \pm 1.3 \mathrm{mV}$ recorded. However, upon amine modification, the surface charge of the PSMI nanoparticles were (in $1 \times$ PBS at pH 7.4) $-32.3 \pm 0.8 \mathrm{mV}$. The reduction in surface charge is due to partial charge neutralisation due to the amine groups. The nanoparticles would however still be stable for the bioconjugation process as particle stability is widely accepted to be associated with surface charges of greater than $\pm 30 \mathrm{mV} .^{\mathbf{1 3}}$

Spectrofluorometry results of the pure reference dye indicated a maximum absorption peak at $569 \mathrm{~nm}$ and a maximum emission peak at $600 \mathrm{~nm}$, as shown in Fig. 5. The position of this maximum emission peak was not altered by encapsulation of the dye into the PSMI nanoparticles, as can be seen from the

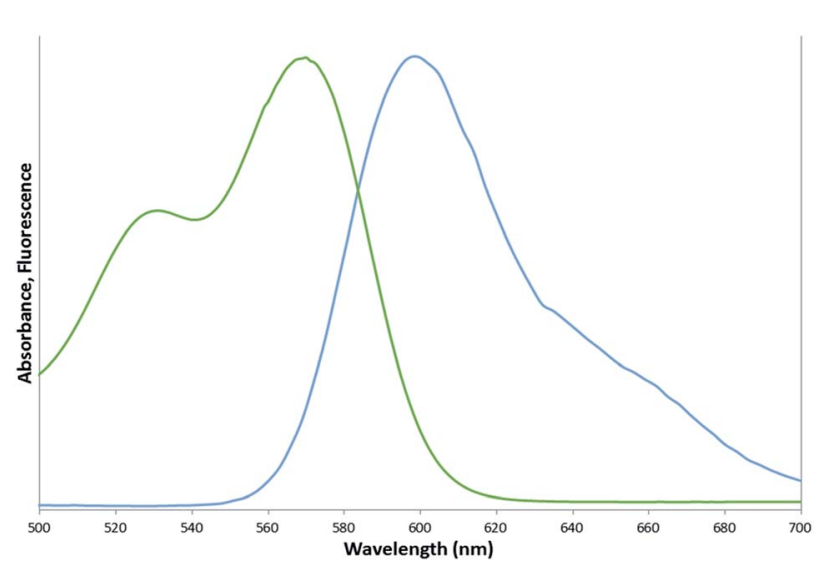

Fig. 5 Maximum absorption and emission profiles for the pure reference dye Exalite 613. 


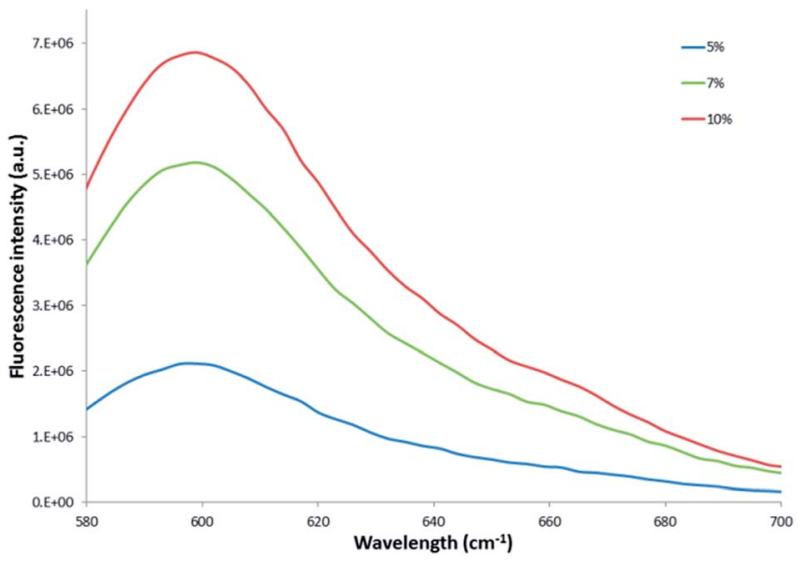

Fig. 6 Comparison of the fluorescence emission intensity of $0.3 \mathrm{mg} \mathrm{mL}^{-1}$ of the $5 \%, 7 \%$ and $10 \%$ dye-loaded nanoparticles, respectively.

emission profiles of dye-doped PSMI nanoparticles shown in Fig. 6.

Comparison of the emission intensity profiles of the $5 \%, 7 \%$ and 10\% dye-loaded nanoparticles (see Fig. 6) indicated that for a similar particle concentration of $0.3 \mathrm{mg} \mathrm{mL}^{-1}$, the $10 \%$ dyeloaded nanoparticles showed the highest emission intensity. Fluorescence emission intensity was measured for serial dilutions of the pure reference Exalite 613 dye as well as for serial dilutions of nanoparticles loaded with $5 \%, 7 \%$ and $10 \%$ dye-loadings. A linear relationship between reference dye concentration and emission intensity was established at reference dye concentrations of $0.1 \mu \mathrm{g} \mathrm{mL}^{-1}$ or lower, as seen in Fig. 7. The three different concentrations of dye-loaded nanoparticles showed linear relationships between nanoparticle concentration and emission intensity at less than 0.4 to $1 \mathrm{mg}$ $\mathrm{mL}^{-1}$, as shown in Fig. 8. The data was fitted with linear regression curves. The emission intensity of an arbitrary concentration of reference dye was compared to that of an equivalent concentration of dye-doped nanoparticles (determined via the weight \% of dye loaded to PSMI polymer during synthesis) using the regression curves fitted to the data shown in Fig. 7 and 8.

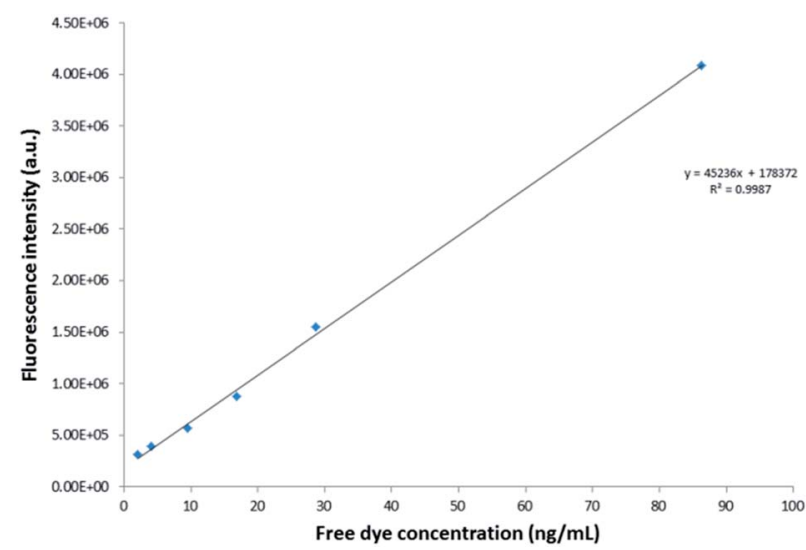

Fig. 7 Relationship between fluorescence intensity and free dye concentration.

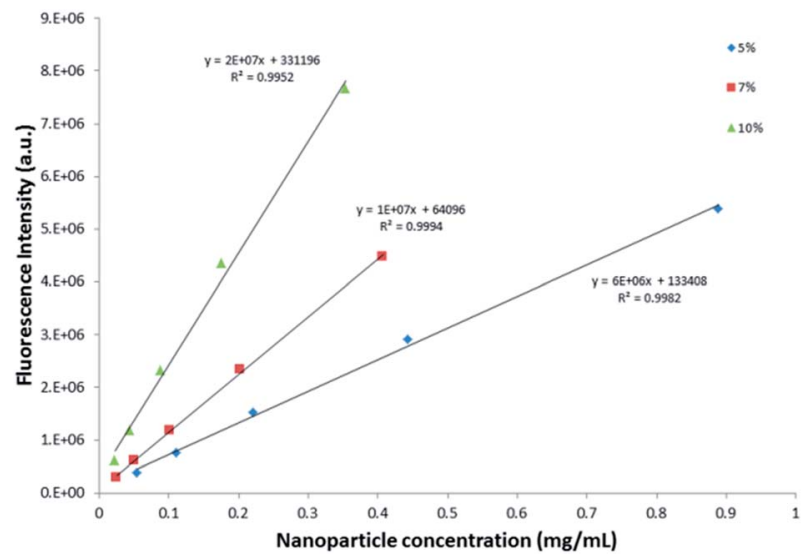

Fig. 8 Relationship between fluorescence intensity and nanoparticle concentration for the $5 \%, 7 \%$ and $10 \%$ dye-loaded nanoparticles, respectively.

Based on these calculations it was concluded that the fluorescence intensity of the encapsulated dye was close to that of the pure reference dye in solution.

Quantification of the immobilized avidin was performed indirectly using a B5F conjugate. ${ }^{12}$ In the presence of avidin, the inherent fluorescence of the B5F conjugate is quenched as a result of the reaction between biotin on the conjugate and avidin in solution. A normalized curve was constructed that indicated the quenching of fluorescence of a specific concentration of B5F associated with a specific avidin concentration (shown in Fig. 9). A linear regression line with descriptive equation was fitted to the normalized curve. The avidin concentration in the supernatant was determined by correlating the maximum amount of fluorescence quenching inflicted on $52.5 \mu \mathrm{L}$ of a $1 \mu \mathrm{g} \mathrm{mL}^{-1}$ solution of the B5F conjugate by different concentrations of nanoparticle supernatant samples to this normalized curve. A volume of $150 \mu \mathrm{L}$ of each point in the dilution series of supernatant was analysed via spectrofluorometry, and the inflection point (point of minimum fluorescent intensity) was taken as the point at which all avidin present in the sample quenched the total concentration of B5F conjugate

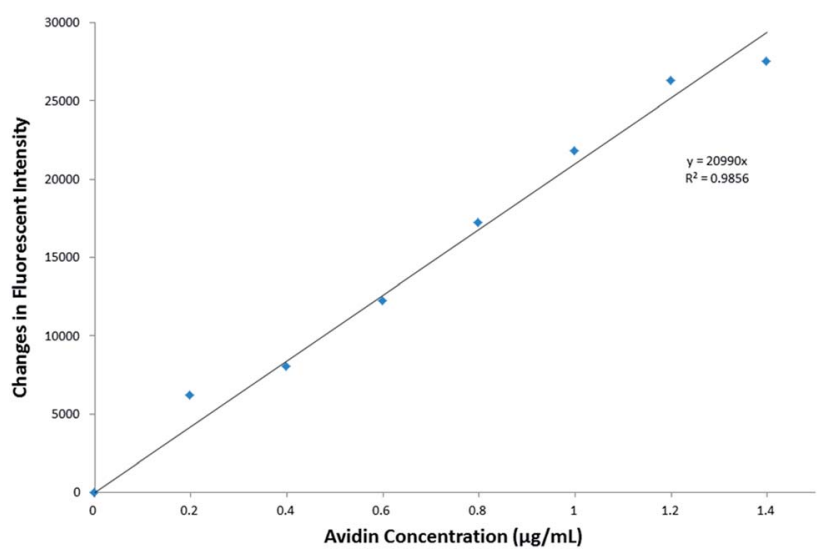

Fig. 9 Avidin standard curve depicting the change in fluorescence of a B5F conjugate resulting from a known concentration of avidin. 
used. ${ }^{14}$ The difference between this minimum fluorescence signal and the fluorescence signal generated by the total volume of $52.5 \mu \mathrm{L}$ of B5F conjugate only was correlated to an avidin concentration via the standard curve shown in Fig. 9. Using this method it was determined that only $6 \%$ of the total avidin added to the nanoparticles was present in the nanoparticle supernatant, and thus not conjugated to the nanoparticle surfaces. This indicates that $94 \%$ of the avidin added to the pure PSMI NPS during bioconjugation was immobilized onto the NP surfaces.

Fixed and sectioned $E$. coli bacteria contacted with anti-E. coli dye-doped PSMI nanoparticles displayed distinctively rougher surfaces when compared to the native $E$. coli not contacted with nanoparticles, as shown in Fig. 10a and b, respectively. The increased surface roughness of the bacteria contacted with nanoparticles could thus be taken as a positive indication of successful binding between nanoparticles and the targeted receptors on the $E$. coli cell walls.

Confocal fluorescence microscopy images of $E$. coli contacted with dye-loaded bioconjugated PSMI nanoparticles and counterstained with SYTO 9 green fluorescent nucleic acid stain are

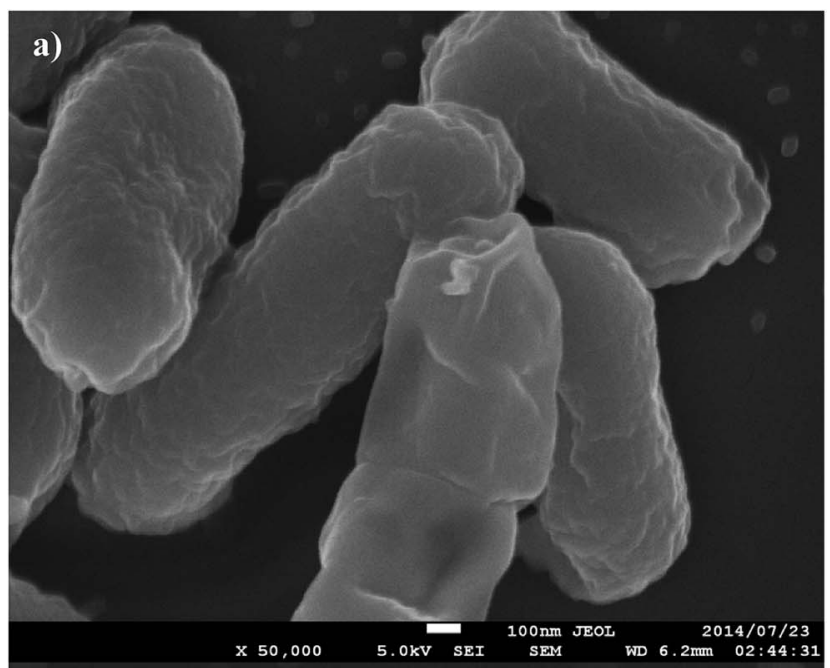

b)

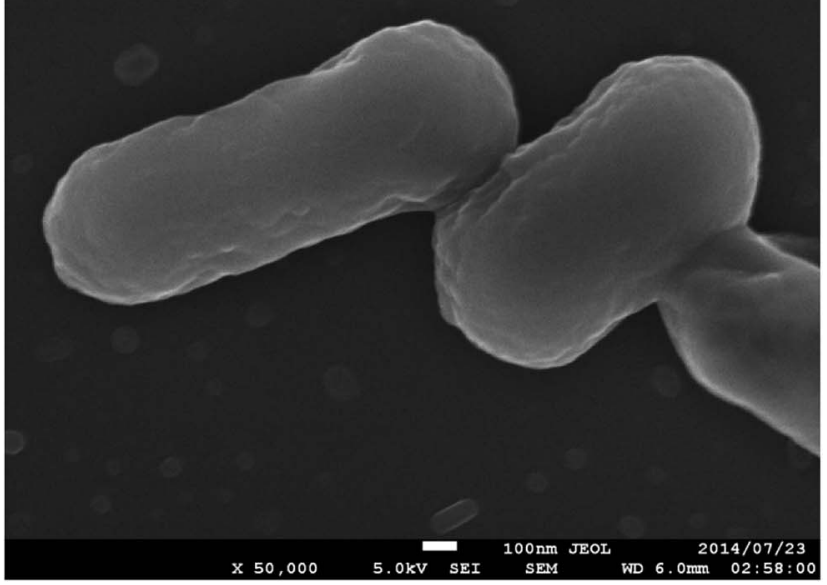

Fig. 10 SEM images of E. coli contacted with dye-doped PSMI nanoparticles conjugated with biotinylated antibodies (a) and control E. coli K-12 bacteria samples (b).

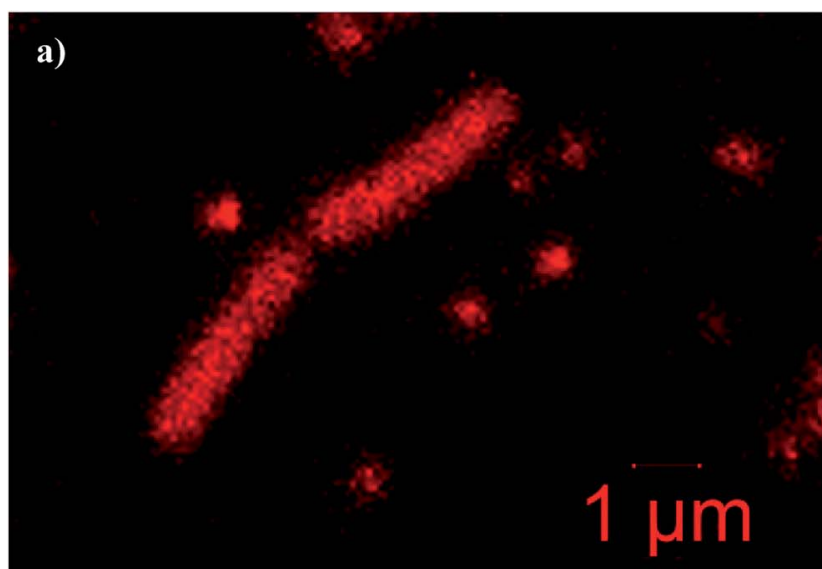

b)

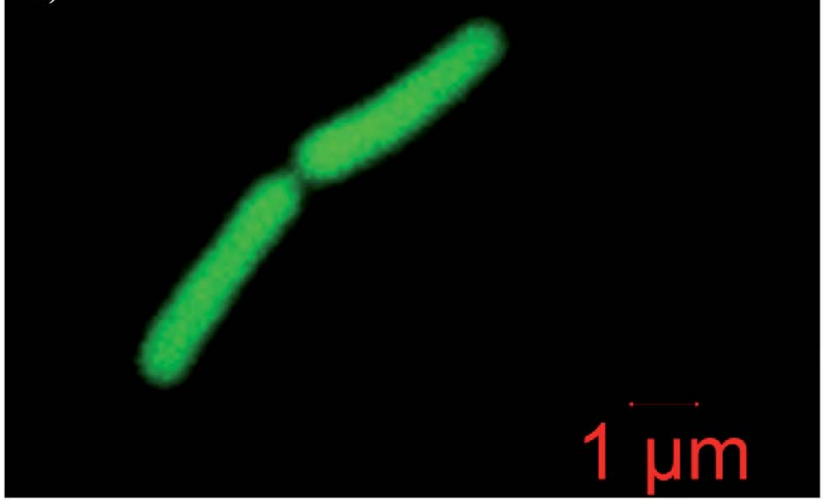

Fig. 11 Confocal fluorescence microgaphs of $E$. coli $\mathrm{K}-12$ cells labelled with (a) dye-loaded bioconjugated PSMI nanoparticles, and (b) counterstained with SYTO 9 nucleic acid stain.

shown in Fig. 11. The E. coli present could be clearly identified by the fluorescent signals generated by the clustering of the anti-E. coli dye-loaded nanoparticles around the individual cells, as demonstrated in Fig. 11a. Fluorescent signals generated by the $E$. coli could be distinguished from background noise by the characteristic size and shape of the cells, and was confirmed by a SYTO 9 counterstain, as shown in Fig. 11b. Noise signals from unbound nanoparticles were expected as an excess of nanoparticles were added to the $E$. coli samples in order to ensure that all cells present would be labelled successfully.

\section{Conclusions}

This contribution describes a simple method for the preparation of dye-doped PSMI nanoparticles and subsequent surface functionalization with amine groups for orientated bioconjugation of antibodies for the selective attachment to E. coli bacteria in aqueous suspensions. Successful imidization of PSMA was confirmed via FT-IR and DSC analyses, and electron microscopy confirmed the formation of nanoparticles with an average size of $50 \mathrm{~nm}$. FT-IR analyses also showed successful surface functionalization of the nanoparticles with amine groups as reactive centres for bioconjugation with avidin. The functionalised nanoparticles displayed increased $T_{\mathrm{g}}$ after surface modification and relatively high zeta-potentials 
showing good particle stability. Fluorescence intensity of the dye-doped nanoparticles increased with dye loading. A B5F titration assay was utilized to determine the efficiency of avidin attachment to the nanoparticles. The avidin conjugated nanoparticles were conjugated with biotinylated anti-E. coli antibodies and successful clustering of dye-doped PSMI nanoparticles around $E$. coli $\mathrm{K}-12$ bacteria was demonstrated. The dye-loaded PSMI nanoparticles thus display the potential to be used as a bright and stable fluorescent probe that can easily be tailored to a specific target via the conjugation of suitable receptor molecules to the nanoparticle surfaces.

\section{Acknowledgements}

The authors would like to acknowledge the following people for their contributions towards this body of work: Khotso Mpitso, Dr Francois Cummings, Dr David Motaung, Antoinette Buys, Charity Maepa and Dr Ben Loos. The authors would also like to thank the Council for Scientific and Industrial Research of South Africa for funding the project. BK wants to acknowledge the support through the South African Research Chairs Initiative (SARChI) from the Department of Science and Technology (DST) and the National Research Foundation (NRF) of South Africa.

\section{Notes and references}

1 X. Zhao, L. R. Hilliard, S. J. Mechery, Y. Wang, R. P. Bagwe, S. Jin and W. Tan, Proc. Natl. Acad. Sci. U. S. A., 2004, 101, 15027-15032.
2 D. Qin, X. He, K. Wang, X. J. Zhao, W. Tan and J. Chen, BioMed Res. Int., 2007, 89364.

3 X. He, L. Zhou, D. He, K. Wang and J. Cao, Analyst, 2011, 136, 4183-4191.

4 Z. Chen, L. Cai, X. Dong, H. Tang and D. Pang, Biosens. Bioelectron., 2012, 37, 75-81.

5 L. Cai, Z. Chen, M. Chen, H. Tang and D. Pang, Biomaterials, 2012, 34, 371-381.

6 R. Edgar, M. McKinstry, J. Hwang, A. B. Oppenheim, R. A. Fekete, G. Giulian, C. Merril, K. Nagashima and S. Adhya, Proc. Natl. Acad. Sci. U. S. A., 2006, 103, 4841-4845.

7 Y. Kuo, Q. Wang, C. Ruengruglikit, H. Yu and Q. Huang, J. Phys. Chem. B, 2008, 112, 4818-4824.

8 X. Xue, J. Pan, H. Xie, J. Wang and S. Zhang, Talanta, 2009, 77, 1808-1813.

9 S. Mazumder, J. Sarkar, R. Dey, M. Mitra, S. Mukherjee and G. Das, J. Exp. Nanosci., 2010, 5, 438-446.

10 A. S. de Dios and M. E. Diaz-Garcia, Anal. Chim. Acta, 2010, 666, 1-22.

11 P. Samyn, M. Deconinck, G. Schoukens, D. Stanssens, L. Vonck and H. Van den Abbeele, Polym. Adv. Technol., 2010, 23, 311-325.

12 Y. Cheng, Y. Liu, J. Huang, K. Li, W. Zhang, Y. Xian and L. Jin, Talanta, 2009, 77, 1332-1336.

13 R. H. Muller, C. Jacobs and O. Kayser, Adv. Drug Delivery Rev., 2001, 47, 3-19.

14 G. Kada, K. Kaiser, H. Falk and H. J. Gruber, Biochim. Biophys. Acta, Gen. Subj., 1999, 1427, 44-48. 